\title{
AN EYE-TRACKING APPROACH TO INATTENTIONAL BLINDNESS
}

\author{
Jennifer M. Pappas \\ Stephanie R. Fishel \\ Jason D. Moss \\ Clemson University - Department of Psychology \\ Clemson, SC \\ Jacob M. Hicks \\ Teri D. Leech \\ Clemson University - Department of Computer Science \\ Clemson, SC
}

\begin{abstract}
Inattentional blindness, the act of failing to notice clearly visible, salient objects in one's environment when engaged in a task, is of great interest due to both its commonality and its overall applications. This study attempted to objectively support previous claims made about the inattentional blindness phenomenon using eye tracking data. It was found that even when a stimulus crossed the fovea, not all individuals saw it. It was also discovered that some participants managed to notice the stimulus without fixating on it, in direct opposition to a hypothesis stating that fixation was required to notice a stimulus.
\end{abstract}

\section{INTRODUCTION}

\section{Inattentional Blindness - A Review}

Inattentional blindness is a psychological phenomenon that has been studied extensively in recent decades. The basic premise is that when individuals are engaging in and attending to a particular task, they are less likely to notice surrounding events, even those that are salient, occurring within their visual field. While the phenomenon has been widely studied, there are still many unknowns. The current study is an attempt to elaborate upon what is already known about inattentional blindness by applying eye tracking technology to a well known paradigm.

The first studies to be conducted on inattentional blindness were in the 1970 s by Neisser $(1975 ; 1979)$. His findings were later extended by Simons and Chabris (1999) who tested inattentional blindness by asking participants to view a video of two teams of participants, one team wearing black and one team wearing white while they were passing a basketball. Depending on the condition, the participants were told by the researchers to count the number of passes made between either the black team or the white team. The researchers used videos with transparent players and a transparent stimulus, as well as videos with opaque players and an opaque stimulus. After approximately 45 seconds, a woman dressed in a black gorilla suit or a woman carrying an umbrella walked across the scene and was visible for 5 seconds. Overall, participants noticed the umbrella woman significantly more (66\%) than they noticed the gorilla (44\%) across all conditions. Also, participants counting the passes made by the black team noticed the gorilla more often than participants counting passes for the white team. This indicated that perhaps similarity of the stimulus (the gorilla) to the attended objects (the black or the white team) had some effect on whether or not the participants noticed the event (Simons \& Chabris, 1999).

In a follow-up study, Most et al, further investigated the role of visual similarity of the stimulus to the attended task objects (2001). They discovered that the stimulus is more likely to be noticed by observers when it is similar to the attended objects. Based on the results of several further studies, the experimenters also concluded that dissimilarity of the stimulus to the ignored set of items has an influence on inattentional blindness.

In addition to the similarity of the stimulus to surrounding objects, researchers have also been interested in the effect of location of the stimulus with respect to the other objects. Newby and Rock (1998) conducted a study examining proximity of the stimulus in relation to inattentional blindness. Their results indicated that inattentional blindness increased in a linear fashion as distance from focal attention increased. Inattentional blindness occurred least (20\%) when the unexpected stimulus was located at focal attention and was greatest when presented at the farthest location from focal attention (60\%).

On a similar note Most, Simons, Scholl, and Chabris conducted a study in an attempt to determine if location of the stimulus had any effect on its detection (2000). Participants focused on the center of a blue line in the center of a display screen and were instructed to count the number of times a black shape ('L' or ' $\mathrm{T}$ ' shaped objects were used, some white and some black) touched the blue line. On the third trial of this nature, an unexpected event occurred, consisting of a grey cross moving in a straight line from right to left across the screen. The cross was on the screen for 5 seconds, and its proximity to the blue center line was varied. More than half (53\%) of observers failed to notice the unexpected cross during this critical trial, even when it passed through the visual focus of attention along the blue line. Participants then 
completed a full attention trial, where they had been notified by an experimenter that an unexpected event might occur. During this full attention trial, the cross was detected by all observers. Another finding of this study was that detection of the unexpected cross decreased the farther away it appeared from the blue horizontal line, supporting the location model of attention.

To summarize, there are several factors that influence the noticing rates of the stimulus in inattentional blindness. As discussed above, both proximity of the stimulus to the attended task as well as feature similarity of the stimulus to the attended task both play crucial roles in whether or not the stimulus is noticed.

\section{Inattentional Blindness and Eye Tracking}

Very few studies have used eye tracking methodology to study inattentional blindness. Caters, Chalmers, and Ledda (2002) looked at inattentional blindness from a computer graphics perspective by asking participants to count the number of pencils in a cup placed in a non-moving computer generated scene. They varied the rendering quality of the background and found that participants were relatively poor at distinguishing the high quality graphics from the low quality graphics. Caters, Chalmers, and Ward (2003) further extended those findings by using two still images with different rendering qualities, one high and one low, and asked participants to count the number of teapots in the scene. Later they were asked to recall any differences between the scenes with regard to quality. Results indicated that $20 \%$ of participants were unable to differentiate between the scene qualities even when eye tracking data confirmed that participants did indeed fixate on objects in the scene that were similar to the teapots (i.e. a vase), supporting the idea that inattentional blindness did indeed occur and not a degraded use of peripheral vision.

Henderson and Hollingworth (2003) looked at inattentional blindness when viewing real-world complex scenes. They were specifically interested in examining whether or not an individual can detect change in a scene if they are fixating on an area where the change takes place. To test this notion, they had two experiments that used a saccadecontingent global display-change paradigm. In both experiments, participants were asked to look at a display consisting of alternating gray and scene vertical strips and to memorize the scene for a future memory test. They were also told to press a button whenever a change in the scene was detected. In experiment 1 , invisible vertical boundaries were placed in such a way as to divide the display into thirds. When the participant crossed the boundaries the scene switched to a slightly different scene. In experiment 2 , the invisible boundaries were placed around three objects in the scene in an aim to determine if participants really did notice the changes in a covert way (i.e. not reported but indicated by fixation durations). Results from experiment 1 indicated that participants were poor at noticing scene changes (detecting only 45 out of 1,691 changes). Experiment 2 revealed similar change detection rates and found no difference in the average fixation durations between the changed and unchanged conditions. The overall results from both experiments indicate that inattentional blindness can occur even when individuals are fixating on the element of a scene that changes. It is also interesting to note that participants did not report the changes overtly, but also failed to noticed the changes covertly.

There are still many questions left unanswered regarding inattentional blindness. Researchers do not know if individuals are missing the stimulus completely or if they perceive the stimulus, but memory fails to encode the information and thus it is forgotten (Mack, 2003; Moore, 2001). Researchers have also studied whether or not inattentional blindness can cause priming, which would indicate implicit perception. It has been found that priming does indeed occur, even when inattentional blindness is demonstrated by an individual. Some believe that stimuli only capture attention when it has some meaning to the individual.

In the present study, eye-tracking technology will be applied to the inattentional blindness paradigm paying particular attention to the similarity and the proximity of the stimulus to the attended objects. A shorter version of the opaque gorilla video used in the Simons and Chabris study (1999) will be presented. Several hypotheses will be tested in the current study. For the purposes of this study, the authors have operationally defined a critical fixation as a significantly longer fixation in the Region of Interest (ROI) indicating detection of the stimulus. It is hypothesized that individuals who see the gorilla will have one significantly longer fixation, a critical fixation, that indicates detection of the stimulus in the ROI, whereas participants who do not see the gorilla will not have a critical fixation. Along these lines, those who notice the stimulus will have a longer total dwell time in the ROI. Also, participants counting passes made by the black team will be more likely to notice the stimulus (i.e. have a critical fixation in the ROI). In addition, the critical fixation for the individuals counting black team passes will occur sooner than the critical fixation for those counting white team passes. Finally, the stimulus is more likely to be noticed when the distance between it and the attended team's basketball is short. Ultimately, this study attempts to confirm the participantive data from previous studies related to inattentional blindness, with objective data collected through eye tracking.

\section{METHODS}

\section{Apparatus}

A Tobii 1750 eye-tracker was used to collect the eye movement data. The display is driven by a dual processor xeon Linux computer and the system is run by a single processor Windows XP computer that sends gaze data over a 100 MBit network to the Linux computer (Tobii User Manual, 2003). The Tobii system is comprised of two screens 
connected to the computer allowing participants to view the stimulus on one screen while the other screen can be used by the experimenter to set up the test and watch the eye-tracking quality during the session. The Tobii eye-tracker finds the participants eyes and calculates gaze positions automatically on the Windows computer allowing the Linux computer to concentrate on displaying the stimulus. A high-resolution camera integrated into a 17 ' TFT display unit with a maximum resolution of $1280 \times 1024$ pixels is used to acquire images of the eyes. The tracker looks like a normal computer. Near infra-red light-emitting diodes are used to capture the reflection patterns on the corneas of the participant's eyes (Tobii User Manual, 2003).

Participants sat approximately $60 \mathrm{~cm}$ away from the display screen which provides the stimulus. The Tobii tracker collects gaze data concurrently for each eye (binocular eyetracking) which leads to higher accuracy. The Tobii eyetracker allows head motion of about $30 \times 15 \times 20 \mathrm{~cm}$ allowing a participant to move their head in a natural manor without having to recalibrate the participant's eyes. The Tobii tracks eye gaze in angles up to +/- 40 degrees from the camera. If the eye-tracker was to lose track of an eye it can recover in less than $100 \mathrm{~ms}$ and automatically resume tracing the eye (Tobii User Manual, 2003). The Tobii eye-tracker has an accuracy of up to 0.5 degrees and an average frame rate of $50 \mathrm{~Hz}$. Typical delay from when the image of the eye is taken and when it is processed is between 25 to $35 \mathrm{~ms}$.

The software used to run the experiment consisted of a video player program and an analysis program. A multimedia player program written using the open source Xine API played the stimuli and used the Tobii eye-tracker data to find the Point of Regard (POR) coordinates on each frame. The analysis program used a velocity threshold algorithm to determine fixations and smooth pursuits.

\section{Stimulus and Participants}

The stimulus was presented in a 31 second video clip consisting of two teams of three participants passing a basketball (one basketball per team) originally used in the Simons and Chabris study (1999) (see Figure 1).

Approximately 15 seconds into the clip the stimulus of interest, a woman dressed in a gorilla suit, walked across the screen from right to left paused in the middle and beat her chest before walking off the screen. The stimulus of interest was visible for approximately 10 seconds.

All participants were undergraduate students at Clemson University and were recruited via word of mouth. There were 19 participants, 10 male and 9 female with a mean age of 19.42 years $(S D=1.43)$.

\section{Experimental Design}

The present study utilized a between participants design. The independent variable was the color of the team the participant was assigned to attend to. The dependent variables of interest were fixation duration in the ROI, total dwell time in the ROI, number of fixations in the ROI, and the time to the critical fixation in the ROI.

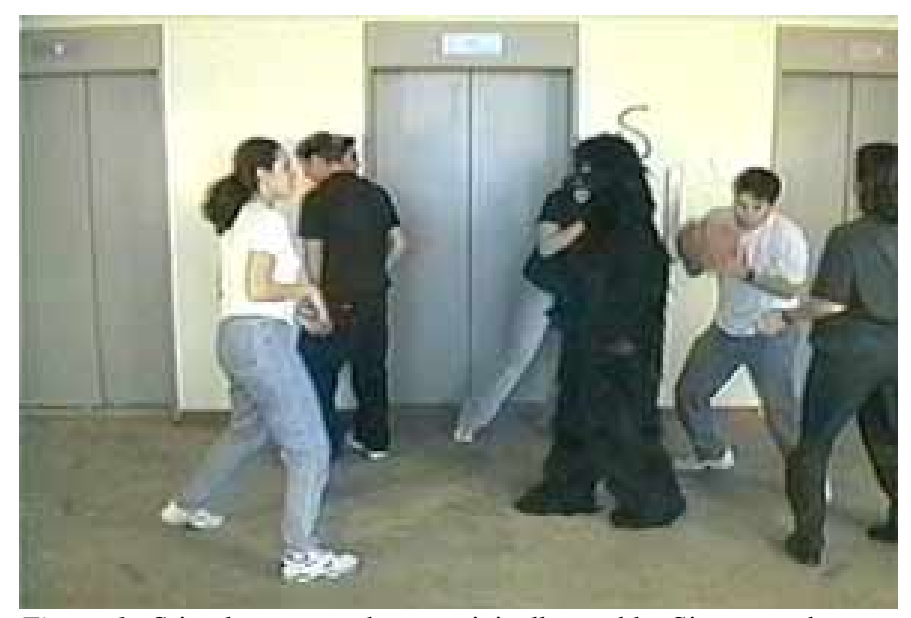

Figure 1. Stimulus screen shot -- originally used by Simons and Chabris (1999).

\section{Procedure}

After arriving at Clemson University's Eye Tracking Laboratory, participants read and completed a Clemson University Institutional Review Board (IRB) approved informed consent form. Participants were then instructed to sit close enough to the computer monitor so that they could see their eyes in the eye tracking portion of the display. Calibration of the eye tracker was completed by asking participants to look at a series of 16 squares that appeared one at a time on the computer monitor. Each square disappeared prior to the appearance of the next square. Upon completion of calibration, participants were asked to view a video clip and were instructed to count the number of passes made by either the team wearing white shirts or black shirts. Team assignments were alternated by participant to keep the groups even.

Participants then completed a questionnaire consisting of the following questions: (i) Which team's passes were you asked to count? (ii) How many passes did you count? (iii) While you were counting, did you notice anything unusual in the video? If yes, please explain. (iv) Did you notice anything other than the six players? If yes, please explain. and (v) Have you previously participated in an experiment similar to this or have you ever heard of such an experiment or the general phenomenon? Following the completion of the experiment the participants were thanked and debriefed.

\section{RESULTS}

\section{Data Reduction and Analysis}

The ROI was designated based on 4 coordinates indicating a rectangle surrounding the gorilla and was, at its maximum, 75 pixels in height and 220 pixels in width. These coordinates were noted for each frame in a file, and the file containing the ROI data was read into the analysis program. 
While creating this file, the frames were evaluated and times when each ball was passing through the ROI were noted based on frame number. The gorilla entered the scene at frame 450 and exited at frame 750, for a total duration of 300 frames. There were 939 frames in the entire video.

The data was analyzed using a hybrid algorithm that determined saccades based on a threshold and the weighted sum of a 5-tap velocity and a 5-tap acceleration filter. The screen distances were measured in degrees visual angle that was computed dynamically at each point based on the number of pixels moved and the participant's actual distance from the screen as measured by the Tobii. Region of Interest (ROI) data was recorded at each frame in the movie as the smallest, axis-aligned rectangle containing the entire gorilla. Total dwell time in the ROI was computed by summing all of the de-noised gaze points contained in the ROI. Total fixation and smooth pursuit time in the ROI was computed by linearly interpolating between the starting and ending point to determine the percentage of each event that was contained in the ROI then taking a sum of that amount. This assumption was validated by the linear nature of most smooth pursuit velocities.

\section{Statistical Tests}

Of the 19 total participants, 2 were not analyzed due to having previously viewed the video, and 3 others were left out due to unusable eye tracking data. Fourteen participants were included in the final data analysis. Though an effort was made to keep the number of participants counting white passes and black passes equal, ultimately 8 participants counted white team passes and 6 participants counted black team passes.

Out of the 14 usable participants, $79 \%$ noticed the stimulus and $21 \%$ did not. This difference in noticing was significant, $X^{2}=4.571, p<.05$. It was hypothesized that the participants assigned to count the black team's passes would be more likely to notice the stimulus due to the color similarity between the team they were concentrating on and the stimulus. This hypothesis was supported, as 100\% (6 out of 6) of the individuals assigned to the black team noticed the gorilla, whereas only 63\% (5 out of 8) of the participants assigned to the white team noticed. This difference between noticing rates approached significance, $X^{2}=2.864, p>.05$. Because of the dynamic nature of the stimulus, there were very few true fixations present in the data after the analysis. Most of the eye movements seen were smooth pursuits, or a cross between a smooth pursuit and a fixation. For the purposes of analysis anything other than a saccade was considered a "fixation".

One of the hypotheses made was that individuals who saw the gorilla would have one significantly longer fixation, a critical fixation, which indicated detection of the stimulus in the ROI, whereas participants who did not see the gorilla would not have a critical fixation. The critical fixation hypothesis was tested by doing a 2 (stimulus seen vs. not seen) x 2 (longest fixation in ROI vs. average of other fixations) mixed factorial ANOVA. In order to support the critical fixation hypothesis, an interaction effect would need to be observed, demonstrating that the presence or absence of a critical fixation depended upon whether or not the gorilla was detected. There was no significant interaction found, $F(1,12)=.779, p>.05$. It is interesting to note that two individuals that did notice the gorilla (18\%) did not have any fixations in the ROI. Also, two of the participants who did not notice the gorilla (67\%), had one or more fixations in the ROI. These exceptions indicate that there is more involved in noticing a stimulus than just having it enter the visual scene, an idea that will be elaborated on later. To this end, the hypothesis that participants counting black team passes would notice the stimulus sooner than the ones counting white team passes is untestable, as there is no way of knowing when the participants actually noticed the stimulus. However, the longest fixation in the ROI for participants counting the black team's passes occurred significantly sooner, at frame 593, than the longest fixation for those counting white team passes, which occurred at frame $675, t(7)=-2.541, p<.05$. This excludes the individuals that had no fixations in the ROI.

Another hypothesis stated that the total dwell time in the ROI would be significantly longer for individuals who noticed the gorilla. There was no significant difference found for total dwell time in the ROI between those who noticed $(M=$ $.638 \mathrm{~s}, S D=.209)$ and those who didn't $(M=.893 \mathrm{~s}, S D=$ .386), $t(12)=-1.081, p>.05$. The final hypothesis was that the stimulus was more likely to be noticed when it was closer to the attended team's basketball. From the location of each team's ball during their fixations (based on frame evaluation conducted during analysis), it was found that $43.8 \%$ of the fixations in the ROI made by participants counting white team passes occurred when the ball or a white team player was in the ROI. Also, $52.8 \%$ of the fixations in the ROI made by the participants counting black team passes occurred when the ball or a black team player was in the ROI. Note that this finding does not indicate that the gorilla was noticed when the ball or player was in the ROI; that information was impossible to gather from the data.

\section{DISCUSSION}

The finding most worthy of discussion deals with the idea of a critical fixation being indicative of whether or not an individual noticed the stimulus. It was expected that individuals who did not notice the gorilla might have some fixations within the ROI, but no critical fixation. But it was not expected for some of the participants who noticed the gorilla to have no fixations at all within the ROI. It is possible that these individuals simply have better peripheral vision or possibly better parallel attention processing abilities. However, it seems more likely that noticing the stimulus does not depend on a critical fixation.

This study does lend support to the ideas of other experimenters that inattentional blindness is a failure of the human brain to encode information. Although it was not focused on in any hypotheses, the fact that two of the 
participants who did not see the gorilla had one or more fixations inside the ROI indicates that, if nothing else, an image of the gorilla was projected onto the retina. It is interesting to find that some participants fail to (1) integrate the information into their visual scene and/or (2) encode this information into memory, especially when it is located near their fovea. Some participants managed to integrate and encode the gorilla when it was clearly in their peripheral vision (e.g., the two participants who did not have any fixations in the ROI). It would be interesting to study whether or not inattentional blindness is related to how observant an individual is on a daily basis. There might be other personality characteristics that specify the type of participants that are likely to be susceptible to the phenomenon.

There are several improvements that can be made on this study. More participants would have given the current results more statistical power. One of the major problems with inattentional blindness studies is that the experimenter cannot create an equal number of participants who notice and participants who do not notice. In this study, the total number of participants was too few; in order to get enough individuals to miss the gorilla, a higher number of overall participants is needed. The noticing rates obtained during this study (21\% of individuals did not notice the gorilla) were comparable to those in previous studies. The difference in noticing the stimulus between the groups counting white and black was already approaching significance with the small number of participants studied; with more participants those numbers would indeed be significant.

With regards to eye tracking, a lot more work with dynamic stimuli needs to be done. There was little literature on smooth pursuit detection and representation. It was also noteworthy that almost no fixations were found in this study, though that could be due to assigning the individuals to watch a moving target the entire time. It would be interesting to repeat the study with another condition where the individuals simply watch the video with no previous knowledge of what they are going to see. This would answer the question of whether or not humans fixate at all during dynamic stimuli. One would expect that the eye movements greatly depend on the objects an individual chooses to attend to in a visual scene.

\section{CONCLUSION}

This study marked the first time objective eye-tracking data was applied to inattentional blindness using a dynamic stimulus. It provided the first objective evidence that inattentional blindness is not simply a result of a failure of the visual perception system. Inattentional blindness appears to be a failure of a cognitive process - perhaps scene integration or memory encoding. Another interesting result of this experiment was the lack of fixations during the presentation of the stimulus. Further eye tracking studies involving dynamic stimuli need to be conducted.

There are many applications for inattentional blindness in human factors, particularly with dynamic stimuli. Driving immediately comes to mind - failure to see a pedestrian enter the road, for example, while changing the music in the car or even while looking directly towards the pedestrian. Future work on inattentional blindness with dynamic stimuli will have far reaching effects by allowing insight to be gained as to why individuals fail to notice salient objects. By understanding why an object is not noticed, we can begin to understand how to make objects noticeable.

\section{ACKNOWLEDGMENTS}

The authors acknowledge Andrew T. Duchowski of Clemson University's Department of Computer Science for his helpful comments on earlier drafts of this paper and allowing us to use the equipment in the eye tracking laboratory.

\section{REFERENCES}

Caters, K., Chalmers, A., Ledda, P. (2002). Selective quality rendering by exploiting human inattentional blindness: Looking but not Seeing. Proceedings of VRST'02, Hong Kong.

Caters, K., Chalmers, A., \& Ward, G. (2003). Detail to attention: Exploiting visual tasks for selective rendering. Eurographics Symposium on Rendering, Leuven, Belgium.

Henderson, J.M. \& Hollingworth, A. (2003). Global transsaccadic change blindness during scene perception. Psychological Science, 14, 493-497.

Mack, A. (2003). Inattentional blindness: Looking without seeing. Current Directions in Psychological Science, 12, 180-184.

Moore, C.M. (2001). Inattentional blindness: Perception or memory and what does it matter. Psyche, 7, NP.

Most, S.B., Simons, D.J., Scholl, B.J., \& Chabris, C.F. (2000). Sustained inattentional blindness: The role of location in the detection of unexpected dynamic events. An Interdisciplinary Journal of Research on Consciousness, 6(14), NP.

Most, S.B., Simons, D.J., Scholl, B.J., Jimenez, R., Clifford, E., \& Chabris, C.F. (2001). How not to be seen: The contribution of similarity and selective ignoring to sustained inattentional blindness. Psychological Science, 12, 9-17.

Neisser, U. (1979). The control of information pickup in selective looking in Perception and its Development: A tribute to Eleanor J Gibson Ed. A D Pick (Hillsdale, NJ: Lawrence Erlbaum Associates), pp 201-219.

Neisser, U., Becklen, R. (1975). Selective looking: Attending to visually specified events. Cognitive Psychology, 7, 480-494.

Newby, E.A., \& Rock, I. (1998). Inattentional blindness as a function of proximity to the focus of attention. Perception, 27, 1025-1040.

Simons, D.J., \& Chabris, C.F. (1999). Gorillas in our midst: Sustained inattentional blindness for dynamic events. Perception, 28, 1059-1074.

Tobii User Manual. (2003.) Tobii, Stockholm, Sweden. http://www.tobii.com/ Version 2.1. 